\title{
Interférences de type Young avec une source à un seul électron
}

François Frémont ${ }^{1}$ (francois.fremont@ensicaen.fr), Anas Hajaji ${ }^{1}$, Raul Oscar Barrachina ${ }^{2}$ et Jean-Yves Chesnel ${ }^{1}$

1- Centre Interdisciplinaire de Recherche Ions Laser, Unité Mixte CEA-CNRS-EnsiCaenUniversité de Caen Basse-Normandie, 6 bd du Maréchal Juin, 14050 Caen Cedex, France 2- Centro Atómico Bariloche et Instituto Balseiro (Comisión Nacional de Energía Atómica et Universidad Nacional de Cuyo), 8400 S. C. de Bariloche, Río Negro, Argentine

Des interférences dues à la diffusion d'un électron unique sur deux protons, qui jouent le rôle de deux trous de Young, ont été mises en évidence expérimentalement pour la première fois. L'électron provient de l'auto-ionisation de l'hélium excité après capture de deux électrons de la molécule $\mathrm{H}_{2}$ par un projectile $\mathrm{He}^{2+}$. Ces interférences

se manifestent par

l'apparition d'oscillations d'intensité dans la distribution angulaire des électrons diffusés. La présence de ces oscillations prouve qu'un électron peut interférer avec lui-même.

L'expérience présente est analogue à une expérience de pensée imaginée par Feynman en 1963.
Nous savons, depuis la fameuse hypothèse de Louis de Broglie en 1924, que la matière peut se comporter soit comme des particules, soit comme des ondes. Le caractère ondulatoire de la matière se manifeste, par exemple, dans les franges (suite de maxima et de minima d'intensité) observées lorsque les objets qui la constituent pénètrent des fentes, ou sont diffractées par un cristal. De nombreuses expériences, réalisées avec des électrons, des atomes, des molécules, des condensats, ont validé cette hypothèse.

Dans le cas particulier des électrons, des expériences ont été consacrées à la mise en évidence d'interférences de type Young. En 1961 par exemple, C. Jönsson a réalisé une vraie expérience d'interférences de ce type, en utilisant un faisceau d'électrons de $50 \mathrm{keV}$ diffusant sur des fentes fines $(\sim 0,3 \mu \mathrm{m})$ et très rapprochées $(\sim 1 \mu \mathrm{m})$ pratiquées dans une feuille de cuivre [1]. Une vingtaine d'années plus tard, deux équipes de chercheurs [2,3] ont réalisé une expérience similaire, en réduisant fortement l'intensité du faisceau incident ; par contre, les auteurs ont fait diffuser des électrons sur un interféromètre qui s'apparente à un biprisme de Fresnel plutôt qu'à un système de fentes.

En 1963, R. Feynman, dans ses célèbres cours [4], met en scène l'expérience suivante : lorsqu'un faisceau d'électrons passe un système interférométrique de type Young (deux fentes), la répartition des électrons sur un écran donne lieu à une alternance de franges lumineuses et sombres, montrant ainsi la nature ondulatoire de l'électron. Que se passe-t-il si l'intensité du faisceau d'électrons est réduite au point qu'un seul électron à la fois passe au travers de deux fentes ? Feynman explique que la répartition des électrons successifs arrivant sur l'écran après traversée des fentes présente aussi des franges d'interférences. Notons que ce résultat indique qu'un électron peut interférer avec lui-même. Cependant, « [Nous ne devons pas] tenter d'effectuer cette expérience. L'expérience n'a jamais été faite de cette façon. La difficulté vient de ce que l'appareil devrait être réalisé à une échelle incroyablement petite pour montrer les effets qui nous intéressent ». Selon les propres termes de Feynman lui-même, ce ne peut être qu'une expérience de pensée.

Résumons les conditions nécessaires à la réalisation de l'expérience de pensée de Feynman : (i) la source doit délivrer un seul électron à la fois ; (ii) l'interféromètre doit être à l'échelle atomique. Les expériences mentionnées ci-dessus ne satisfont pas toutes ces conditions. Par exemple, le flux d'électrons dans l'expérience de Jönsson [1] est tel que la condition (i) d'électron unique n'est pas respectée. Dans les expériences [2,3], même si l'intensité du faisceau est considérablement réduite, rien n'indique que la condition (i) est atteinte.

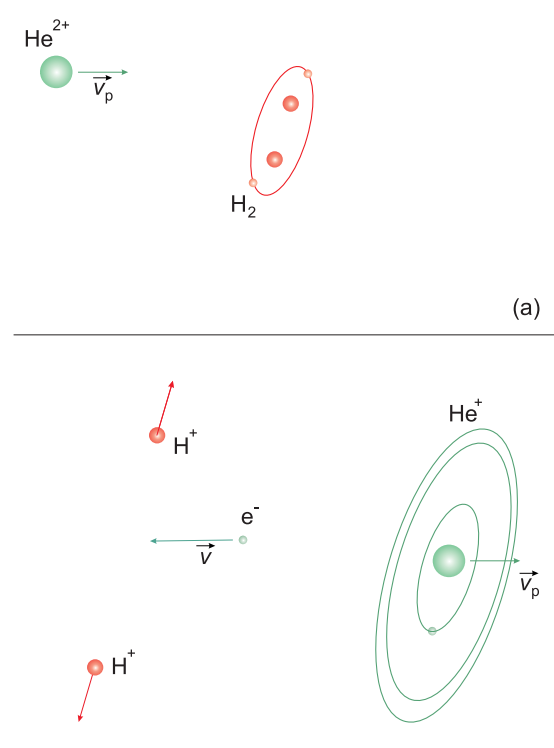

(b)

Figure 1 : Scénario de la collision utilisée afin de réaliser l'expérience d'interférences de type Young avec une source à électron unique. Un ion $\mathrm{He}^{2+}$ s'approche, à basse énergie, d'une cible moléculaire $\mathrm{H}_{2}$ (a). L'ion capture les deux électrons de $\mathrm{H}_{2}$ et se retrouve dans un état excité. La cible, ayant perdu ses deux électrons, se dissocie, donnant lieu à deux protons. Le projectile perd un électron par effet Auger, cet électron étant émis, a priori, dans une direction quelconque. S'il est émis vers l'arrière (b), donc vers les protons, l'électron diffuse sur eux, donnant lieu à des interférences qui se manifestent par l'apparition de franges alternativement sombres et claires. Les deux grandes ellipses autour du noyau d'He représentent de façon schématique les orbitales des deux électrons capturés dans l'état excité de l'atome d'He. 


\section{Réalisation d'une expérience d'interférences de type Young}

Il y a un peu plus d'un an, nous avons finalement réalisé [5] une expérience très proche de l'expérience de pensée de Feynman. Proposé et étudié théoriquement en 2004 [6], le principe de l'expérience est le suivant : un ion incident $\mathrm{He}^{2+}$ passe au voisinage d'une cible moléculaire $\mathrm{H}_{2}$ (fig. 1a). Dans des temps voisins de $10^{-15} \mathrm{~s}$, l'ion $\mathrm{He}^{2+}$ capture, avec une forte probabilité, les deux électrons de $\mathrm{H}_{2}$. Après la collision, le projectile $\mathrm{He}$ est dans un état très excité, alors que la cible $\mathrm{H}_{2}{ }^{2+}$ se dissocie, donnant lieu à des protons $\mathrm{H}^{+}$.

Le projectile $\mathrm{He}$ se désexcite principalement par effet Auger, au bout d'un temps de l'ordre de $10^{-13} \mathrm{~s}$ : un des deux électrons revient sur un état plus fondamental, donnant son excédent d'énergie au second électron qui est alors éjecté avec une énergie d'environ $35 \mathrm{eV}$, correspondant à une vitesse d'émission $v_{e}$ d'environ $3,510^{6} \mathrm{~m} / \mathrm{s}$, dans le référentiel lié au projectile. Lorsque l'émission a lieu vers l'arrière par rapport à la direction incidente $\mathrm{du}$ projectile, donc vers les protons, l'électron éjecté diffuse sur les deux $\mathrm{H}^{+}$ (fig. 1b) qui jouent le rôle de deux trous d'Young séparés de seulement quelques $\mathrm{nm}$. La condition (ii) est ainsi réalisée. Le résultat de cette diffusion est l'apparition de franges d'interférences, dont la période angulaire est d'environ $20^{\circ}$. Les auteurs [6] ont montré que la condition (i) d'électron unique est bien respectée. En effet, le projectile n'émet qu'un seul électron, et l'interféromètre $\left(\mathrm{H}^{+}-\mathrm{H}^{+}\right)$est détruit après le passage de l'électron, puisque les deux $\mathrm{H}^{+}$s'éloignent l'un de l'autre. De plus, l'intensité du faisceau primaire d'hélium et

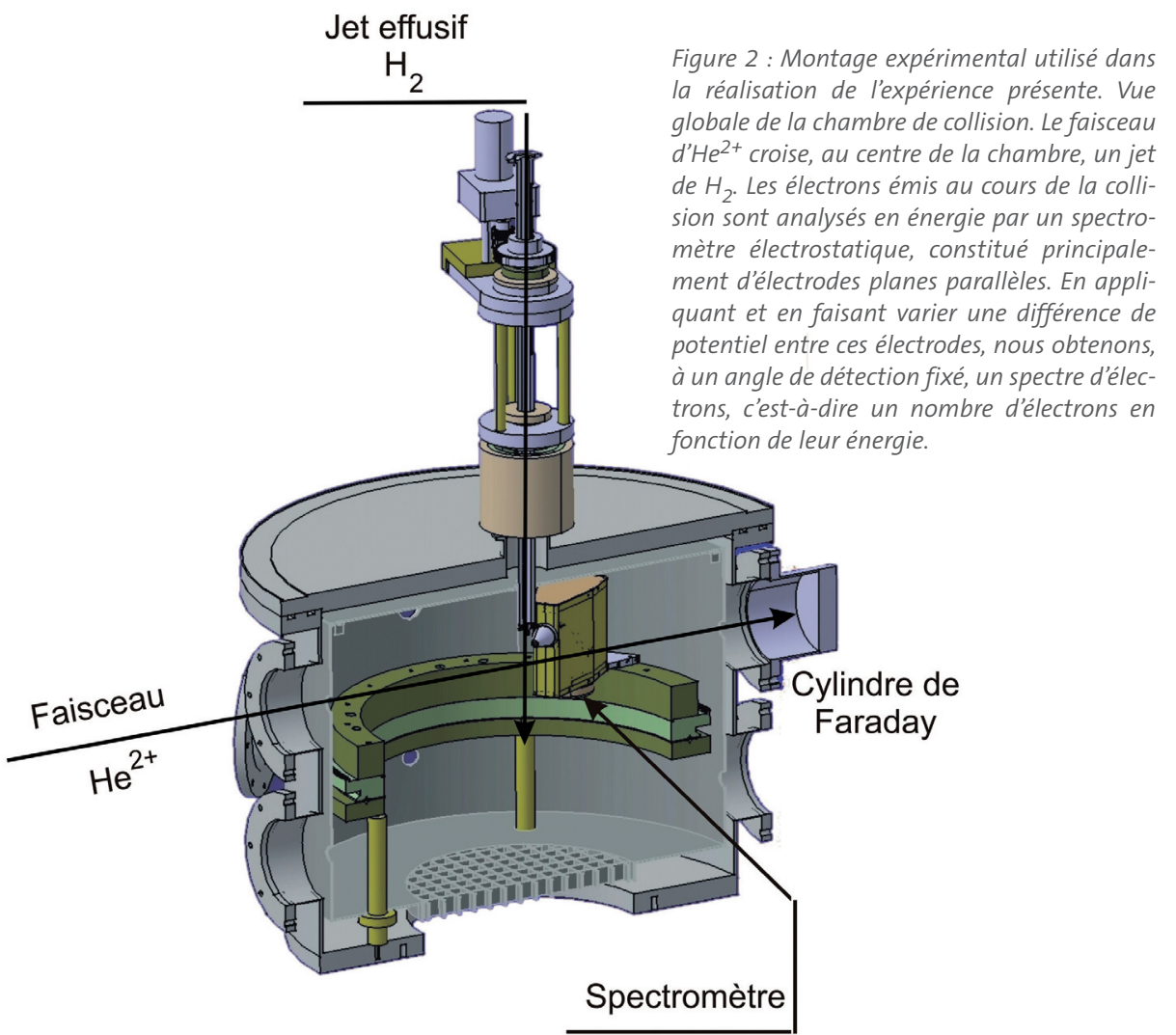

la densité de gaz sont suffisamment faibles pour que les événements soient bien séparés les uns des autres. Autrement dit, l'expérience proposée est une accumulation d'expériences individuelles mettant en jeu, après un événement, une nouvelle source à électron unique et un nouvel interféromètre.

\section{Montage expérimental}

Cette expérience a été réalisée sur la ligne ARIBE (Accélérateurs pour les Recherches avec des Ions de Basse Énergie) du GANIL (Grand Accélérateur National d'Ions Lourds) à Caen. Le faisceau $\mathrm{d}^{\prime} \mathrm{He}^{2+}$, produit par une source de type RCE (Résonance Cyclotron Électronique), est extrait à des énergies de $8 \mathrm{keV}$ et $30 \mathrm{keV}$, autrement dit à des vitesses respectives de $0,710^{6} \mathrm{~m} / \mathrm{s}$ et $1,410^{6} \mathrm{~m} / \mathrm{s}$, et est focalisé dans une chambre de collision (fig. 2), avec un diamètre de quelques $\mathrm{mm}$. Le courant d'ions collecté dans le cylindre de Faraday à la sortie de la chambre est typiquement de $100 \mathrm{nA}$, correspondant à une densité de projectiles de $\sim 10^{5}$ ions par $\mathrm{cm}^{3}$. Au centre de la chambre, le faisceau de ces projectiles croise une cible constituée d'un jet effusif de gaz $\mathrm{H}_{2}$. La pression du gaz dans cette cible est de l'ordre de $10^{-4} \mathrm{mbar}$, ce qui donne une densité de $\sim 10^{11}$ molécules par $\mathrm{cm}^{3}$. Sous ces conditions, nous avons montré [5] que le rapport $p_{2} /\left(p_{1}\right)^{2}$ est inférieur à $10^{-12}$, où $p_{2}$ est la
Figure 3 : Spectres typiques d'électrons émis dans la collision $\mathrm{He}^{2+}+\mathrm{H}_{2}$, à une énergie de $30 \mathrm{keV}$ et à des angles de détection de $160^{\circ}$ (a) et $120^{\circ}$ (b). Les structures (1), (2) et (3) proviennent de la désexcitation par effet Auger du projectile, après une double capture dans des états très excités du projectile. La courbe en traits discontinus ajuste la contribution de l'ionisation directe.
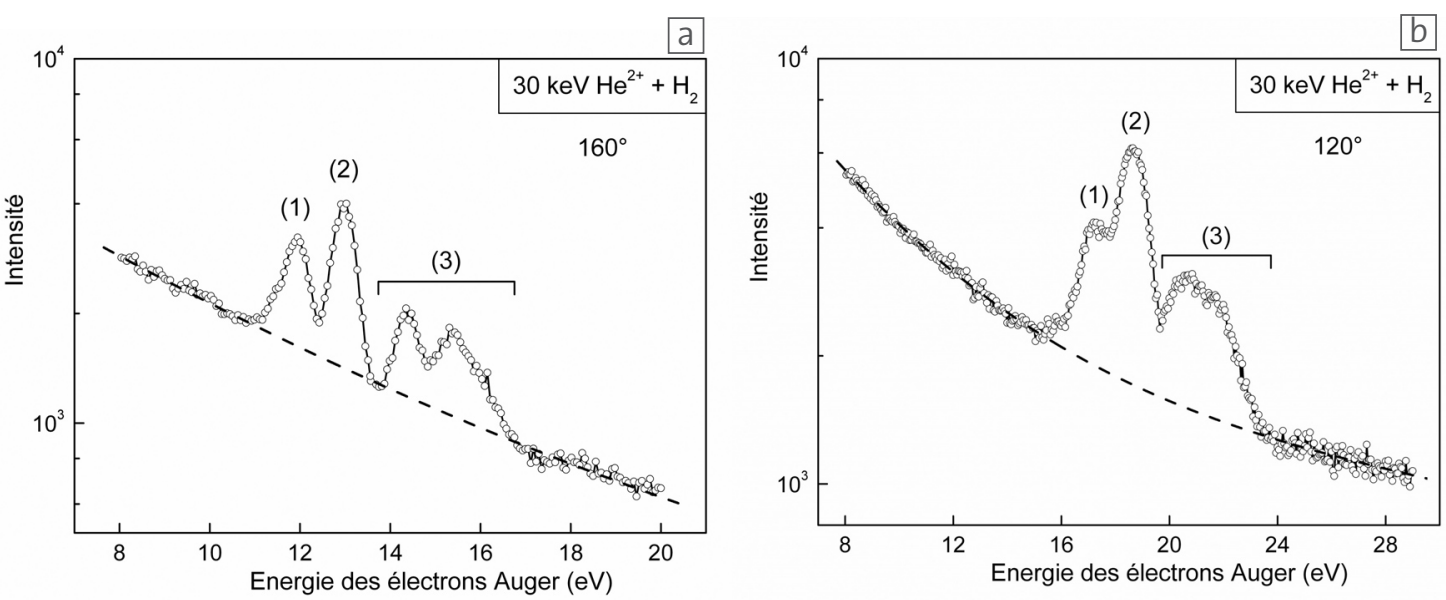
Figure 4 : Nombre d'électrons Auger émis après une double capture dans la collision $\mathrm{He}^{2+}+\mathrm{H}_{2}$, à une énergie de $30 \mathrm{keV}$, en fonction de l'angle de détection. Aux angles avant $\left(20^{\circ}-90^{\circ}\right)$, aucune oscillation n'est observée. En revanche, aux angles arrière, c'est-à-dire entre $90^{\circ}$ et $160^{\circ}$, trois oscillations au moins sont clairement visibles, superposées à une contribution croissante avec l'angle. Elles sont dues aux interférences causées par la diffusion d'un électron sur les deux protons, qui servent de trous de Young. La période angulaire $\sim 17^{\circ}$ est celle prédite par la théorie [6].

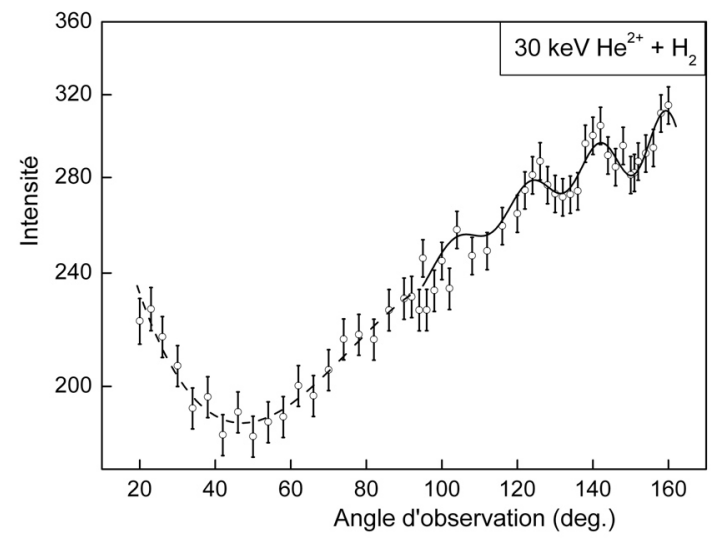

probabilité que deux électrons provenant de deux projectiles différents diffusent sur le même interféromètre $\left(\mathrm{H}^{+}-\mathrm{H}^{+}\right)$, et $p_{1}$ est la probabilité qu'un seul électron atteigne un interféromètre. L'inégalité $p_{2} /\left(p_{1}\right)^{2}<<1$, qui a déjà été utilisée dans des expériences d'interférences mettant en jeu une source de photon unique [7], assure que la condition d'électron unique est bien respectée.

Les électrons produits après la collision sont détectés et analysés en énergie par un spectromètre électrostatique. En appliquant et en faisant varier une différence de potentiel entre les électrodes planes de ce spectromètre, un spectre d'électrons est obtenu, consistant en un nombre d'électrons en fonction de leur déviation angulaire, qui est directement liée à leur énergie. Cette distribution est obtenue en faisant varier l'angle de détection, défini par rapport à la direction incidente du faisceau d'hélium, entre $20^{\circ}$ et $160^{\circ}$.

\section{Spectres :}

\section{analyse et discussion}

\section{Exemples de spectres}

Sur la figure $3 \mathrm{a}$ (voir page précédente) est présenté un spectre typique, obtenu pour une énergie de projectile de $30 \mathrm{keV}$ et un angle de détection de $160^{\circ}$. Deux contributions sont clairement séparées :

- la partie monotone, ajustée au moyen d'un polynôme en échelle log-log (tirets sur la figure 3a), provient de l'éjection d'un électron de $\mathrm{H}_{2}$ par interaction directe entre le projectile et cet électron. Son intensité décroît fortement lorsque l'énergie de l'électron émis croît. Ce processus est appelé ionisation directe ;

- les structures qui se détachent de la partie monotone sont dues à la désexcitation du projectile par effet Auger, après la capture des deux électrons de $\mathrm{H}_{2}$ (voir ci-dessus).
Les structures, notées (1), (2) et (3), correspondent respectivement à des configurations du projectile de nombres quantiques différents.

La distribution angulaire des électrons émis a été obtenue par intégration en énergie des pics d'auto-ionisation. Cependant, comme le montre la figure $3 b$ (p. 11), lorsque l'angle de détection diminue, la largeur des pics augmente considérablement. Les structures (1) et (2) ne peuvent donc plus être séparées. Pour cette raison, la distribution angulaire a été obtenue pour le spectre d'auto-ionisation dans sa globalité. Néanmoins, il a été montré théoriquement [6] que cela n'affecte pas le résultat final sur les interférences. Seule une diminution du contraste de la figure d'interférences est attendue.
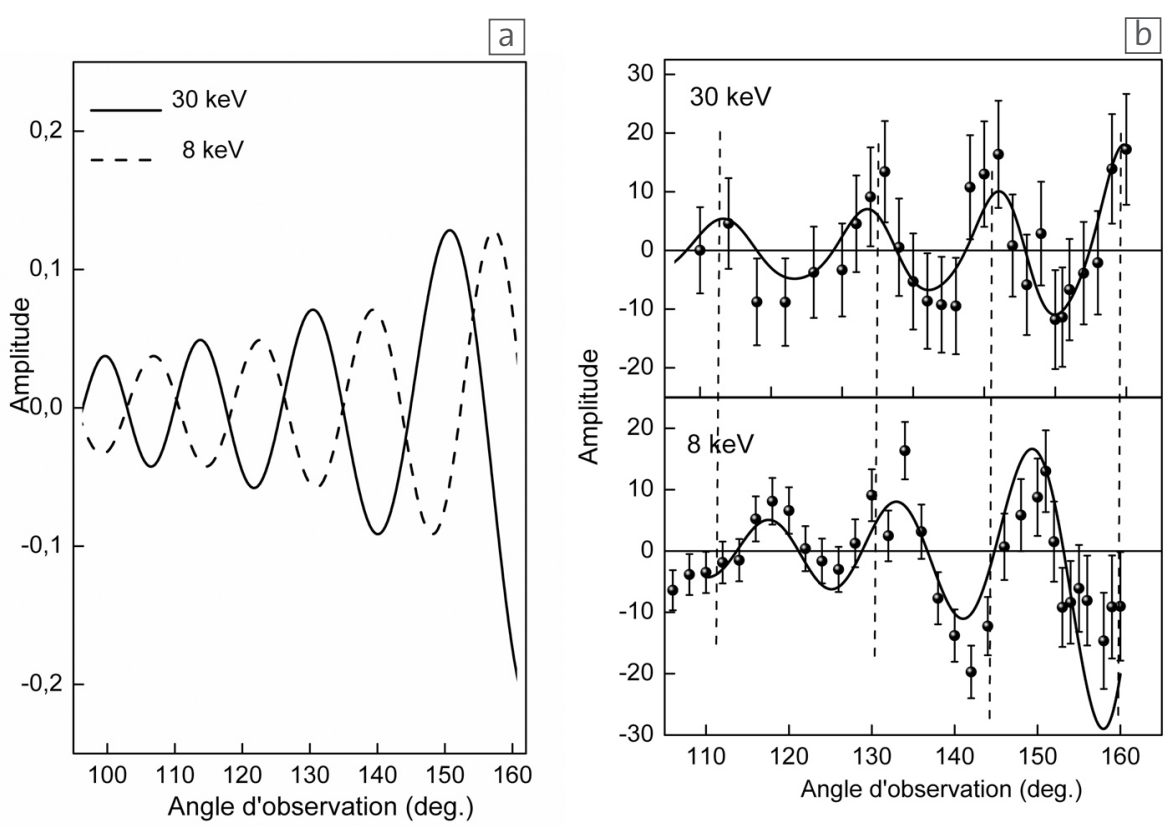

Figure 5: Oscillations prédites (a) par le modèle théorique [6] lorsque l'énergie du projectile est de $30 \mathrm{keV}$ (courbe en traits pleins) et $8 \mathrm{keV}$ (courbe en traits discontinus), donc lorsque la vitesse du projectile est diminuée d'un facteur 2 . Les oscillations sont toujours présentes mais, dans le domaine d'observation $\left(110^{\circ}-160^{\circ}\right)$, un déphasage de $\pi$ est attendu. L'expérience réalisée (b) confirme la prédiction théorique. Sur la figure $5 b$, la contribution croissante avec l'angle (cf. fig. 4) a été retranchée. 
de la lumière par une pupille circulaire, l'amplitude du terme d'oscillation décroît au fur et à mesure que l'on s'éloigne du centre de l'écran, correspondant à un angle de détection de $180^{\circ}$. L'amplitude dépend, outre de l'angle de détection, de la distance $d$ entre les protons, et de la vitesse $\vec{v}$ de l'électron Auger dans le référentiel $\mathrm{du}$ laboratoire. À $30 \mathrm{keV}$, cette vitesse est $v=v_{e}-v_{p}=2,110^{6} \mathrm{~m} / \mathrm{s}$ à un angle de détection de $180^{\circ}$. Lorsque l'énergie (et donc la vitesse) $\mathrm{du}$ projectile diminue, $v$ augmente, ce qui a pour conséquence de diminuer la longueur d'onde associée de l'électron émis. Une diminution de la vitesse du projectile fait donc varier le terme d'oscillation. On peut montrer que l'effet de la diminution de la vitesse du projectile d'un facteur 2 est, dans la gamme angulaire $90^{\circ}-160^{\circ}$, un déphasage de $\pi$ (fig. 5a). C'est exactement ce que nous trouvons expérimentalement (fig. 5b), lorsque l'énergie du projectile passe de $30 \mathrm{keV}$ (haut de la figure 5b) à $8 \mathrm{keV}$ (bas de la figure 5b).

\section{Conclusion}

Le présent travail est la première réalisation de l'expérience de pensée de Feynman. Nous avons montré qu'un électron unique qui diffuse sur deux centres $\mathrm{H}^{+}$, jouant le rôle de deux trous de Young, interfere avec lui-même. Les conditions requises pour la réalisation d'une telle expérience de pensée ont été remplies : la source n'émet qu'un seul électron avant d'être remplacée par une autre source, un seul électron à la fois atteint l'interféromètre, et l'interféromètre est à l'échelle atomique.

Par la suite, nous allons étudier les interférences produites lors des collisions $\mathrm{He}^{2+}+\mathrm{H}_{2}$ à plus basse énergie de projectile. Aux énergies les plus basses disponibles au GANIL, de l'ordre de $50 \mathrm{eV}$, la vitesse du projectile est environ $410^{4} \mathrm{~m} / \mathrm{s}$. À ces vitesses, le projectile passe suffisamment de temps près des deux protons pour perturber leur trajectoire et modifier considérablement leur vitesse finale. Dans ce cas, la répartition angulaire des électrons émis peut être sensiblement modifiée, à cause du changement de la distance entre les protons lorsque l'électron est émis. Nous nous attendons, par exemple, à ce que la période des oscillations diminue avec la vitesse du projectile, ce qui n'est pas le cas aux énergies de projectile déjà explorées.

Sur le plan théorique, de nombreuses questions restent ouvertes. Pour le moment, le modèle théorique repose sur plusieurs approximations. En particulier, dans ce modèle, la distance $d$ entre les protons, ainsi que le temps au bout duquel l'électron est émis, ne sont que des valeurs moyennes. Nous savons, par exemple, que l'électron Auger est émis dans un domaine de temps très large, avec une probabilité qui décroît exponentiellement avec le temps. De plus, le modèle [6] prévoit que $d$ dépend de l'instant d'émission de l'électron. Par conséquent, chaque événement implique une distance donnée, mais quelconque, entre les protons. Puisqu'à chaque distance correspond une période d'oscillations, nous nous attendons à ce que la distribution angulaire résulte de la superposition d'un grand nombre d'oscillations de périodes différentes. Or, expérimentalement, une seule période angulaire semble se dégager $\left(\sim 17^{\circ}\right)$. L'amélioration du modèle est donc cruciale pour une compréhension détaillée de ce phénomène d'interférences.

\section{Références}

1 C. Jönsson, Z. Phys. 161 (1961) 454 ; traduction anglaise : C. Jönsson, Am. J. Phys. 42 (1974) 4.

2. P.G. Merli, G.F. Missiroli et G. Pozzi, Am. J. Phys. 44 (1976) 306.

3. A. Tonomura, J. Endo, T. Matsuda, T. Kawasaki et H. Ezawa, Am. J. Phys. 57 (1989) 117.

4- R. Feynman, R.B. Leighton et M. Sands, The Feynman Lectures on Physics (Reading MA: Addison-Wesley, 1963) vol. 3, chapitre 37.

5- J.-Y. Chesnel, A. Hajaji, R.O. Barrachina et F. Frémont, Phys. Rev. Lett. 98 (2007) 100403.

6- R.O. Barrachina et M. Zitnik, J. Phys. B 37 (2004) 3847.

7. V. Jacques, E. Wu, T. Toury, F. Treussart, A. Aspect, P. Grangier et J.-F. Roch, Eur. Phys. J. D 35 (2005) 561. 